\title{
Commentary: Feedback stabilizes propagation of synchronous spiking in cortical neural networks
}

\author{
Enric Claverol-Tinturé ${ }^{1 *}$ and Guenter Gross ${ }^{2}$ \\ ${ }^{1}$ Catalonia Foundation for Science and Innovation, Barcelona, Spain, ${ }^{2}$ Center for Network Neuroscience, University of North \\ Texas, Denton, TX, USA
}

Keywords: spiking neuronal networks, feedback, timing, brain evolution, oscillations, synchronization

\section{A commentary on}

Feedback stabilizes propagation of synchronous spiking in cortical neural networks by Moldakarimov, S., Bazhenov, M., and Sejnowski, T. J. (2015). Proc. Natl. Acad. Sci. U.S.A. 112, 2545-2550. doi: 10.1073/pnas.1500643112

Recently published computational work (Moldakarimov et al., 2015) investigated local network feedback as a physiologically plausible strategy for the preservation of precise spike timing in propagating volleys.

Since the timing of spikes contributes to conveying and processing information across species, information modalities and brain areas (Singer, 1999; Jermakowicz and Casagrande, 2007; Harvey et al., 2013; Zuo et al., 2015), it is reasonable to expect that nature has developed strategies to retain, when needed, narrow spike-time spreads in population activity as it propagates along multi-synaptic paths.

Indeed, it has been demonstrated computationally that weak stimuli and the resulting sparse volleys are at risk of propagation failure and desynchronization as activity traverses multiple neuronal layers (Diesmann et al., 1999; Vogels and Abbott, 2005). Moldakarimov and colleagues show that feedback can do the trick and preserve spike timing even in weak propagating volleys. They first simulated a 5-layer purely feedforward network with Hodgkin-Huxley conductance cell models and observed how weak stimulation of layer 1 did not succeed in reaching layer 5 with similar intensity and low jitter. However, adding feedback connections from layer $\mathrm{N}$ to layer $\mathrm{N}-1$, targeting both excitatory and inhibitory cells, succeeds in propagating sparse activity with narrow timing spreads.

These results merit consideration from an evolutionary perspective in the context of the wider problem of brain scalability. Throughout evolution brain mass increased and neuronal aggregates reorganized (Clark et al., 2001; Herculano-Houzel et al., 2007, 2014). Remarkably, many anatomically and functionally distinctive modules in the mammalian brain have been preserved and enlarged by evolution, partially substantiating the hypothesized scalability of brain's architectural plan (Clark et al., 2001). Yet much remains to be understood regarding the computational advantages of brain size, the limits of scalability and its compatibility with the biophysical constraints imposed by neuronal tissue.

One such biophysical constraint stems from the finite conduction velocity of axons (Swadlow et al., 1978). Bigger brains with longer propagation paths and increased end-to-end delays could hamper the synchronization of activity among distant areas. Instead, oscillatory dynamics with isochronous discharges do occur over widely distributed areas, irrespective of brain size (Roelfsema et al., 1997; Varela et al., 2001; Uhlhaas et al., 2009). 
Various compensatory mechanisms have been proposed to counter delays and support scalability. Buzsáky and colleagues hypothesized that a subpopulation of heavily myelinated axons with larger than average diameters might be eventually discovered where and when faster conduction velocities and moderate time lags are needed (Buzsáki et al., 2013). An alternative, dynamic relaying, proposes that intermediate populations linked by recurrent connections to distant areas can induce their synchronization despite relatively large time lags (Vicente et al., 2008; Barardi et al., 2014; Gollo et al., 2014).

Prioritizing first spike timing to spike rates as information carrier over afferent tracks could also confer scalability. Consider for example the human visual system, capable of categorizing images within an astonishing $100 \mathrm{~ms}$, as demonstrated by local field potentials in the inferior occipital gyrus recorded from epileptic patients undergoing surgery (Liu et al., 2009; Tang et al., 2014).

It stands to reason that the enlargement of the brain throughout evolution likely resulted in the flow of visual information from the retina necessarily traveling longer distances to reach high-level cortical areas. How could the visual system be scalable and robust against growing delays? Perhaps favoring first spike timing as information carrier played a role (Thorpe et al., 1996; Gutig et al., 2013). Single spike processing could be fast and more resilient against brain-size related delays than spike rate computation because the latter requires the observation of relatively long epochs of spike trains. With first spike computation fast image categorization could then be retained despite increasing brain size and scalability and robustness against delays might ensue.

It is against this background of ongoing research on the limits of brain scalability related to brain size that the work by Moldakarimov and colleagues can be seen as relevant to the broader endeavor of understanding brain evolution. They

\section{References}

Abeles, M. (1982). Local Cortical Circuits. An Electrophysiological Study. Berlin; Heidelberg: Springer.

Barardi, A., Sancristobal, B., and Garcia-Ojalvo, J. (2014). Phase-coherence transitions and communication in the gamma range between delaycoupled neuronal populations. PLoS Comput. Biol. 10:e1003723. doi: 10.1371/journal.pcbi.1003723

Buzsáki, G., Logothetis, N., and Singer, W. (2013). Scaling brain size, keeping timing: evolutionary preservation of brain rhythms. Neuron $80,751-764$. doi: 10.1016/j.neuron.2013.10.002

Clark, D. A., Mitra, P. P., and Wang, S. S. (2001). Scalable architecture in mammalian brains. Nature 411, 189-193. doi: 10.1038/350 75564

Devor, A., Bandettini, P. A., Boas, D. A., Bower, J. M., Buxton, R. B., Cohen, L. B., et al. (2013). The challenge of connecting the dots in the B.R.A.I.N. Neuron 80, 270-274. doi: 10.1016/j.neuron.2013.09.008

Diesmann, M., Gewaltig, M. O., and Aertsen, A. (1999). Stable propagation of synchronous spiking in cortical neural networks. Nature 402, 529-533. doi: $10.1038 / 990101$

Eberle, A. L., Selchow, O., Thaler, M., Zeidler, D., and Kirmse, R. (2015). Mission (im)possible - mapping the brain becomes a reality. Microscopy 64, 45-55. doi: 10.1093/jmicro/dfu104 explore feedback as a compensatory mechanism that counters undesired intra-volley desynchronisation which could arise as new tissue and synaptic layers were added by evolution. Their work adds to very relevant research on synfire chains (Abeles, 1982; Jahnke et al., 2013) often approaching also the problem of desynchronization of volleys.

In the coming years it is likely that the emerging connectome will shed light on the subject elucidating further compensatory mechanisms developed by evolution. A myriad of techniques including Diffusion Tensor Imaging (DTI), automated 3D reconstruction from optical and electron microscopy data, fMRI, EEG, MEG, and others, are increasingly supporting a concerted effort for systematic whole-brain anatomical and functional mapping of brain connectivity (Devor et al., 2013; Van Essen, 2013; Hale, 2014; Mitra, 2014; Eberle et al., 2015). Connections undergoing evolution-driven elongation, potentially increased time lags, as well as structures with multilayer cytoarchitectures at risk of desynchronizing weak volleys as new layers are added, will be systematically identified. The hypothesized compensatory strategies, being increased myelination and thicker axons, local feedback connections, dynamic relay architectures or others will be eventually confirmed or refuted.

Computational neuroscientists are uniquely positioned to tackle the grand challenge of understanding brain evolution. Simulations can efficiently validate the functional advantages of presumed evolutionary events and the correct sequence in which these took place. The work of Moldakarimov and colleagues contributes toward solving this puzzle and motivates further research.

\section{Acknowledgments}

The authors would like to thank John E. Chad and Jerry Pine for useful discussions.
Gollo, L. L., Mirasso, C., Sporns, O., and Breakspear, M. (2014). Mechanisms of zero-lag synchronization in cortical motifs. PLoS Comput. Biol. 10:e1003548. doi: 10.1371/journal.pcbi.1003548

Gutig, R., Gollisch, T., Sompolinsky, H., and Meister, M. (2013). Computing complex visual features with retinal spike times. PLOS ONE 8:e53063. doi: 10.1371/journal.pone.0053063

Hale, M. E. (2014). Mapping circuits beyond the models: integrating connectomics and comparative neuroscience. Neuron 83, 1256-1258. doi: 10.1016/j.neuron.2014.08.032

Harvey, M. A., Saal, H. P., Dammann, J. F. 3rd., and Bensmaia, S. J. (2013). Multiplexing stimulus information through rate and temporal codes in primate somatosensory cortex. PLoS Biol. 11:e1001558. doi: 10.1371/journal.pbio. 1001558

Herculano-Houzel, S., Collins, C. E., Wong, P., and Kaas, J. H. (2007). Cellular scaling rules for primate brains. Proc. Natl. Acad. Sci. U.S.A. 104, 3562-3567. doi: 10.1073/pnas.0611396104

Herculano-Houzel, S., Manger, P., and Kaas, J. H. (2014). Brain scaling in mammalian evolution as a consequence of concerted and mosaic changes in numbers of neurons and average neuronal cell size. Front. Neuroanatomy 8:77. doi: 10.3389/fnana.2014.00077

Jahnke, S., Memmesheimer, R. M., and Timme, M. (2013). Propagating synchrony in feed-forward networks. Front. Comput. Neurosci. 7:153. doi: 10.3389 /fncom.2013.00153 
Jermakowicz, W. J., and Casagrande, V. A. (2007). Neural networks a century after Cajal. Brain Res. Rev. 55, 264-284. doi: 10.1016/j.brainresrev.2007.06.003

Liu, H., Agam, Y., Madsen, J. R., and Kreiman, G. (2009). Timing, timing, timing: fast decoding of object information from intracranial field potentials in human visual cortex. Neuron 62, 281-290. doi: 10.1016/j.neuron.2009.02.025

Mitra, P. P. (2014). The circuit architecture of whole brains at the mesoscopic scale. Neuron 83, 1273-1283. doi: 10.1016/j.neuron.2014.08.055

Moldakarimov, S., Bazhenov, M., and Sejnowski, T. J. (2015). Feedback stabilizes propagation of synchronous spiking in cortical neural networks. Proc. Natl. Acad. Sci. U.S.A. 112, 2545-2550. doi: 10.1073/pnas.1500643112

Roelfsema, P. R., Engel, A. K., Konig, P., and Singer, W. (1997). Visuomotor integration is associated with zero time-lag synchronization among cortical areas. Nature 385, 157-161. doi: 10.1038/385157a0

Singer, W. (1999). Neuronal synchrony: a versatile code for the definition of relations? Neuron 24, 111-125.

Swadlow, H. A., Rosene, D. L., and Waxman, S. G. (1978). Characteristics of interhemispheric impulse conduction between prelunate gyri of the rhesus monkey. Exp. Brain Res. 33, 455-467.

Tang, H., Buia, C., Madhavan, R., Crone, N. E., Madsen, J. R., Anderson, W. S., et al. (2014). Spatiotemporal dynamics underlying object completion in human ventral visual cortex. Neuron 83, 736-748. doi: 10.1016/j.neuron.2014. 06.017

Thorpe, S., Fize, D., and Marlot, C. (1996). Speed of processing in the human visual system. Nature 381, 520-522. doi: 10.1038/381520a0

Uhlhaas, P. J., Pipa, G., Lima, B., Melloni, L., Neuenschwander, S., Nikolic, D., et al. (2009). Neural synchrony in cortical networks: history, concept and current status. Front. Integr. Neurosci. 3:17. doi: 10.3389/neuro.07.017.2009
Van Essen, D. C. (2013). Cartography and connectomes. Neuron 80, 775-790. doi: 10.1016/j.neuron.2013.10.027

Varela, F., Lachaux, J. P., Rodriguez, E., and Martinerie, J. (2001). The brainweb: phase synchronization and large-scale integration. Nat. Rev. Neurosci. 2, 229-239. doi: 10.1038/35067550

Vicente, R., Gollo, L. L., Mirasso, C. R., Fischer, I., and Pipa, G. (2008). Dynamical relaying can yield zero time lag neuronal synchrony despite long conduction delays. Proc. Natl. Acad. Sci. U.S.A. 105, 17157-17162. doi: 10.1073/pnas.0809353105

Vogels, T. P., and Abbott, L. F. (2005). Signal propagation and logic gating in networks of integrate-and-fire neurons. J. Neurosci. 25, 10786-10795. doi: 10.1523/jneurosci.3508-05.2005

Zuo, Y., Safaai, H., Notaro, G., Mazzoni, A., Panzeri, S., and Diamond, M. E. (2015). Complementary contributions of spike timing and spike rate to perceptual decisions in rat s1 and s2 cortex. Curr. Biol. 25, 357-363. doi: 10.1016/j.cub.2014.11.065

Conflict of Interest Statement: The authors declare that the research was conducted in the absence of any commercial or financial relationships that could be construed as a potential conflict of interest.

Copyright (C) 2015 Claverol-Tinturé and Gross. This is an open-access article distributed under the terms of the Creative Commons Attribution License (CC BY). The use, distribution or reproduction in other forums is permitted, provided the original author(s) or licensor are credited and that the original publication in this journal is cited, in accordance with accepted academic practice. No use, distribution or reproduction is permitted which does not comply with these terms. 